\title{
Probing parity doubling in nucleons at high temperature
}

\author{
Gert Aarts $^{a}$, Chris Allton ${ }^{\ddagger a, b \dagger}$, Simon Hands ${ }^{a}$, Benjamin Jäger ${ }^{a}$, Chrisanthi Praki $^{a}$, \\ Jon-Ivar Skullerud ${ }^{c, d}$ \\ ${ }^{a}$ Department of Physics, College of Science, Swansea University, Swansea SA2 8PP, United \\ Kingdom
}

${ }^{b}$ All Souls College, Oxford OXI 4AL, United Kingdom

${ }^{c}$ Department of Mathematical Physics, National University of Ireland Maynooth, Maynooth, County Kildare, Ireland

${ }^{d}$ School of Mathematics, Trinity College, Dublin 2, Ireland

$\dagger$ E-mail: c.allton@swan.ac.uk

\begin{abstract}
The spectrum of nucleons and their parity partners is studied as a function of temperature spanning the deconfinement transition. We analyse our results using the correlation functions directly, exponential fits in the hadronic phase, and the Maximum Entropy Method. These techniques all indicate that there is degeneracy in the parity partners' channels in the deconfined phase. This is in accordance with the expectation that there is parity doubling and chiral symmetry in the deconfined phase. In the hadronic phase, we also find that the nucleon ground state is largely independent of temperature, whereas there are substantial temperature effects in the negative parity channel. All results are obtained using our FASTSUM 2+1 flavour ensembles.
\end{abstract}

The 33rd International Symposium on Lattice Field Theory

14 - 18 July 2015

Kobe International Conference Center, Kobe, Japan*

\footnotetext{
*Speaker.
} 


\section{Introduction}

Chiral symmetry in the mesonic sector has been extensively studied at non-zero temperature [1] but, due to the extra level of difficulty, there have been few such studies in the baryonic sector. On the lattice, baryonic analyses have been confined to a quenched study of parity doubling [3] and screening mass studies at both zero [2,3] and non-zero density [4]. The theoretical expectation is that when chiral symmetry is restored (as occurs above the deconfining temperature, $T_{c}$ ) parity partners in the baryon sector become degenerate.

In nature, at zero temperature (where chiral symmetry is broken) the positive parity nucleon state has mass $M_{N}=939 \mathrm{MeV}$ whereas its negative parity partner has a mass of $M_{N *}=1535 \mathrm{MeV}$. The status of the parity channels above $T_{c}$ is not yet clear from experiment, but since protons are routinely measured in heavy-ion collisions, it is of great importance to study the pattern of parity doubling theoretically.

In this talk we study full QCD lattice nucleon correlators and their parity properties using a variety of techniques for temperatures in both hadronic and plasma phases. Full results appear in [5], but we extend this work by including new Maximum Entropy Method (MEM) analyses [6].

\section{Nucleon Correlation Functions}

We use one of the standard interpolation operators for the nucleon, i.e.

$$
O_{N}(\mathbf{x}, \tau)=\varepsilon_{a b c} u_{a}(\mathbf{x}, \tau)\left[u_{b}^{T}(\mathbf{x}, \tau) \mathscr{C} \gamma_{5} d_{c}(\mathbf{x}, \tau)\right]
$$

where $u, d$ are the quark fields, $a, b, c$ are colour indices, other indices are suppressed and $\mathscr{C}$ denotes the charge conjugation matrix. We can define positive and negative parity operators and their corresponding zero momentum correlators as

$$
O_{N_{ \pm}}(\mathbf{x}, \tau)=P_{ \pm} O_{N}(\mathbf{x}, \tau), \quad G_{ \pm}(\tau)=\int d^{3} x\left\langle\operatorname{Tr} O_{N_{ \pm}}(\mathbf{x}, \tau) \bar{O}_{N_{ \pm}}(\mathbf{0}, 0)\right\rangle
$$

where the parity projector is $P_{ \pm}=\frac{1}{2}\left(1 \pm \gamma_{4}\right)$ and the trace indicates the sum over Dirac indices, see [5]. For systems with chiral symmetry, a chiral rotation on the quark fields shows that the two parity channels are degenerate, and, up to overall minus signs [7],

$$
G_{ \pm}(\tau)=G_{\mp}(\tau)=G_{ \pm}(1 / T-\tau),
$$

where the temporal extent of the lattice is the inverse temperature, $N_{\tau} a_{\tau}=1 / T$. Using time reflection properties, $G_{ \pm}(\tau)$ contains both parity channels each propagating either forwards or backwards in time. For this reason we consider $G_{+}(\tau)$ only and denote this as $G(\tau)$ from now on.

\section{Lattice details}

We use our FASTSUM $2+1$ flavour, anisotropic ensembles with the same parameters as used by the Hadron Spectrum Collaboration [8]. The details of our lattice parameters are listed in Table 1. Our light quarks correspond to $M_{\pi}=384(4) \mathrm{MeV}$, with $M_{\pi} / M_{\rho}=0.466(3)$; the strange quark 


\begin{tabular}{cccccc}
\hline$N_{s}$ & $N_{\tau}$ & $T[\mathrm{MeV}]$ & $T / T_{c}$ & $N_{\text {cfg }}$ & $N_{\text {src }}$ \\
\hline 24 & 128 & 44 & 0.24 & 171 & 2 \\
24 & 40 & 141 & 0.76 & 301 & 4 \\
24 & 36 & 156 & 0.84 & 252 & 4 \\
24 & 32 & 176 & 0.95 & 1000 & 2 \\
24 & 28 & 201 & 1.09 & 501 & 4 \\
24 & 24 & 235 & 1.27 & 1001 & 2 \\
24 & 20 & 281 & 1.52 & 1000 & 2 \\
24 & 16 & 352 & 1.90 & 1001 & 2 \\
\hline
\end{tabular}

Table 1: Details of the ensembles. The lattice size is $N_{s}^{3} \times N_{\tau}$, with the temperature $T=1 /\left(a_{\tau} N_{\tau}\right) . N_{\text {cfg }}$ $\left(N_{\text {src }}\right)$ denotes the number of configurations (sources) used at each volume. The spatial lattice spacing is $a_{s}=0.1227(8) \mathrm{fm}$, with anisotropy $a_{s} / a_{\tau}=3.5$.

mass is tuned to the physical value [9]; and $T_{c}$ is determined via the renormalised Polyakov loop. Details of these ensembles are available in Refs. [10-12].

We use Gaussian smearing for the sources and sinks [13] tuned to give the best signal for the positive parity ground state at the lowest temperature. Our links are APE-smeared and we use Chroma software [14] to calculate the correlation functions. Details of our procedures are in [5].

\section{Results}

The nucleon correlators are shown in the left pane of Fig. 1 as a function of $\tau T$ for the various temperatures. In our case, the $+(-)$ parity states propagate forwards(backwards) in time. The data points corresponding to the minimum of $G(\tau)$ within errors are highlighted with a shaded rectangle. As can be seen, for $T \lesssim T_{c}$ this minimum region is clearly not at the central point, $\tau T=\frac{1}{2}$, whereas for $T \gtrsim T_{c}$ it is. Therefore, even from direct studies of the correlators without any fitting procedure, there is evidence that the parity partners in the nucleon spectrum become degenerate for $T \gtrsim T_{C}$.

This analysis is further underlined in right panes of Fig. 1 where correlators for the hottest and coldest temperatures are shown separately for the forward (+ve parity) and time-reflected backward (-ve parity) parts of the correlators. The (identical) lines in both graphs are a guide to the eye and again show clear parity breaking at low temperatures and indicate parity doubling at high temperatures.

To study the onset of parity doubling, we define the ratio of correlation functions [3],

$$
R(\tau)=\frac{G(\tau)-G(1 / T-\tau)}{G(\tau)+G(1 / T-\tau)}
$$

Note that at the central point, $R\left(\frac{1}{2 T}\right)=0$ since $R(1 / T-\tau)=-R(\tau) . R(\tau)$ is naturally between 0 and 1: in the highly non-degenerate case where the +ve and -ve parity masses satisfy $M_{+} \ll M_{-}$ we have $R(\tau) \approx 1$ (for $\tau \neq \frac{1}{2 T}$ ), whereas in the degenerate case, we have $R(\tau) \equiv 0$. The results for the ratio are displayed in the left pane of Fig. 2 showing that $R(\tau) \neq 0$ in the hadronic phase, $T<T_{c}$, indicating the absence of parity doubling. (The drop towards zero as $\tau \rightarrow \frac{1}{2 T}$ is unavoidable 

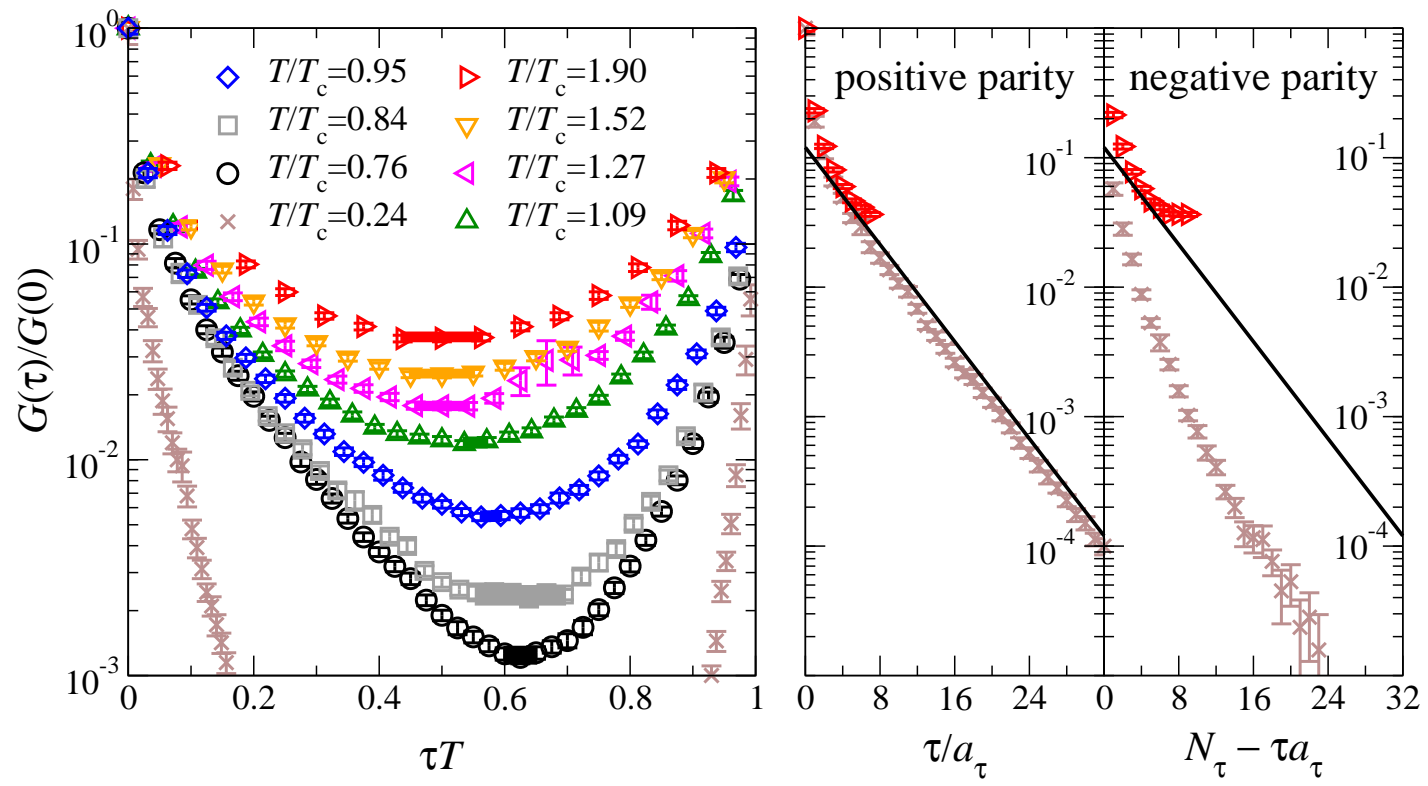

Figure 1: Left pane: Euclidean correlator $G(\tau) / G(0)$ as a function of $\tau T$. At each temperature, the filled rectangle indicates where the correlator is a minimum within errors. Right panes: The same ratio but versus $\tau / a_{\tau}$ and for the hottest and coldest temperatures only. The positive and negative parity channels are shown separately. The two (identical) guide lines indicate parity non-degeneracy for low $T$ and degeneracy at high $T$.

as explained above.) As the temperature increases, the ratio decreases and is very close to zero for $T \gtrsim T_{c}$, signally parity doubling.

In order to quantify parity degeneracy further, we consider the average ratio $R$, defined as

$$
R=\frac{\sum_{n=1}^{\frac{1}{2} N_{\tau}-1} R\left(\tau_{n}\right) / \sigma^{2}\left(\tau_{n}\right)}{\sum_{n=1}^{\frac{1}{2} N_{\tau}-1} 1 / \sigma^{2}\left(\tau_{n}\right)},
$$

where $\sigma(\tau)$ is the statistical error in $R(\tau)$ and $\tau_{n}=n a_{\tau}$. Again, $R \approx 1$ signals a parity broken phase and $R \approx 0$ a parity-doubling phase. Figure 2 (right pane) plots $R$ showing a clear crossover behaviour matching our earlier findings of parity doubling at $T \approx T_{c}$.

For $T<T_{c}$, we fit the correlators to standard exponentials obtaining the masses shown in Table 2 which agree with the results from [15] obtained using variational fitting techniques. At the lowest temperature, these masses are higher than in nature, but this is presumably due to our quarks being unphysically heavy $[9,15]$. From the fit results, $M_{+}$has very little temperature dependence whereas $M_{-}$does. Above $T_{c}$, exponential fits are found to be unreliable, presumably due to the ground states no longer being well-defined particles. We include in the table the dimensionless ratio,

$$
\Delta=\frac{M_{-}-M_{+}}{M_{-}+M_{+}}
$$

to quantify the onset of parity doubling. In nature, $\Delta=0.241$ at $T=0$, and $\Delta \equiv 0$ in the paritydegenerate phase. The table shows that we agree with nature's value at low $T$ (within errors) 

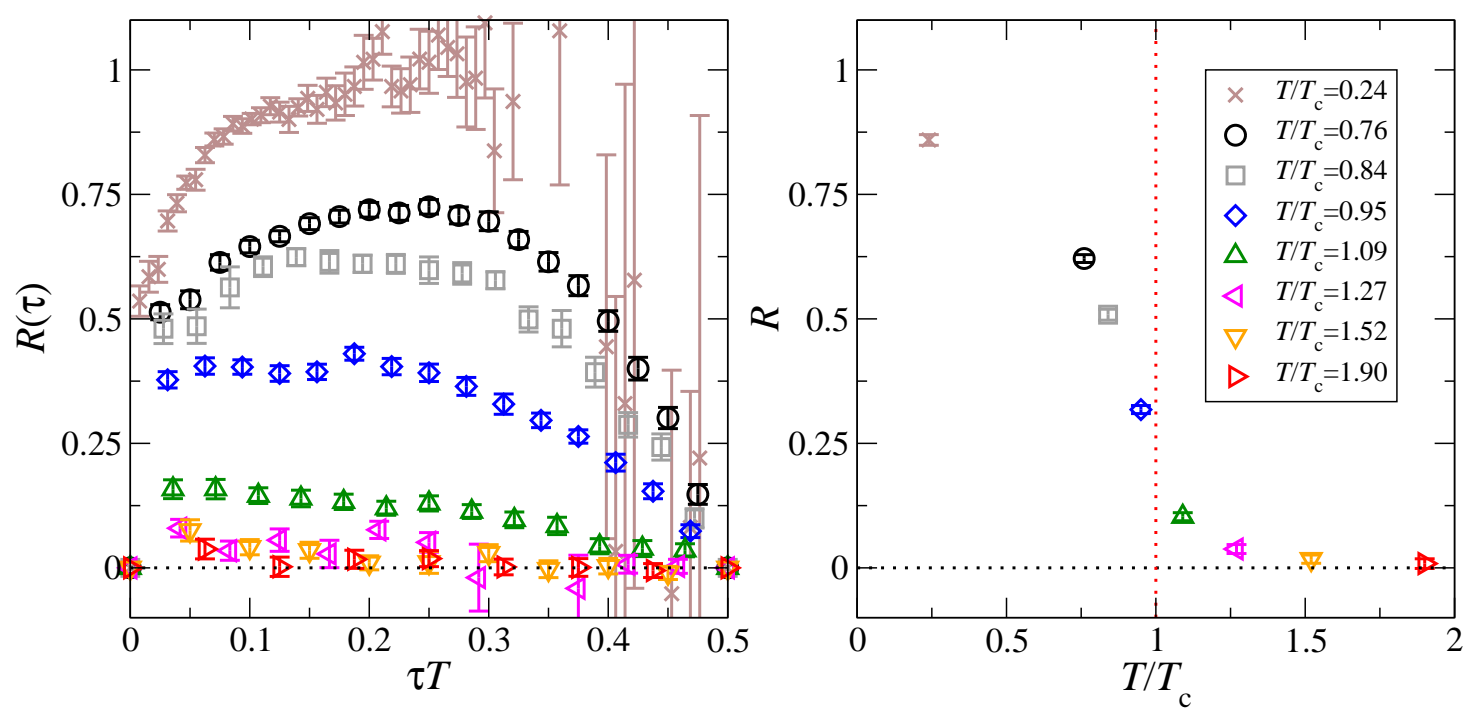

Figure 2: Left pane: The Ratio $R(\tau)$ in Eq. 4.1 as a function of $\tau T$. Right pane: Averaged ratio $R$ defined in Eq. 4.2 as a function of $T / T_{c}$.

\begin{tabular}{cccccc}
\hline$T / T_{c}$ & $a_{\tau} M_{+}$ & $a_{\tau} M_{-}$ & $M_{+}[\mathrm{GeV}]$ & $M_{-}[\mathrm{GeV}]$ & $\Delta$ \\
\hline 0.24 & $0.213(5)$ & $0.33(5)$ & $1.20(3)$ & $1.9(3)$ & $0.209(28)(082)$ \\
0.76 & $0.209(16)$ & $0.28(3)$ & $1.18(9)$ & $1.6(2)$ & $0.138(29)(130)$ \\
0.84 & $0.192(17)$ & $0.28(2)$ & $1.08(9)$ & $1.6(1)$ & $0.197(39)(054)$ \\
0.95 & $0.198(25)$ & $0.22(4)$ & $1.12(14)$ & $1.3(2)$ & $0.052(35)(190)$ \\
\hline
\end{tabular}

Table 2: Results from standard exponential fits to the correlators below $T_{c}$ with statistical and systematic errors added in quadrature. The final column contains $\Delta$ (see Eq. 4.3) with estimates of statistical and systematic errors shown separately. In nature at $T=0, \Delta=0.241$.

and that $\Delta$ then reduces, albeit within large systematic errors due to the difficulty of performing exponential fits in the negative parity sector at high temperature.

Further analysis can be made by spectrally decomposing the correlator $G(\tau)$,

$$
G(\tau)=\int_{0}^{\infty} \frac{d \omega}{2 \pi}\left[\frac{e^{-\omega \tau}}{1+e^{-\omega \tau}} \rho_{+}(\omega)-\frac{e^{-\omega(1 / T-\tau)}}{1+e^{-\omega \tau}} \rho_{-}(\omega)\right],
$$

see [16] for details. We can extract the spectral functions $\rho_{ \pm}(\omega)$ for the \pm ve parity states from the MEM [17] using an extended integration domain and a modified kernel, $K(\omega, T)$,

$$
\begin{aligned}
& G(\tau) \equiv \int_{-\infty}^{+\infty} \frac{d \omega}{2 \pi} K(\tau, \omega) \rho(\omega) \quad \text { and } \quad \rho_{ \pm}(\omega) \equiv \rho(\omega) \quad \text { for } \quad \omega \gtrless 0, \\
& \text { where } \quad K(\tau, \omega)=\frac{e^{-\omega \tau}}{1+e^{-\omega / T}} \quad \omega>0 \\
& =\frac{e^{+\omega(1 / T-\tau)}}{1+e^{+\omega / T}} \quad \omega<0 .
\end{aligned}
$$


Details of this procedure will appear shortly [6], but we show our main results in Fig. 3 where the spectral functions are plotted for various temperatures. The MEM analysis confirms the above picture of a parity non-degenerate phase for $T \lesssim T_{c}$ with parity doubling, i.e. $\rho_{+}(\omega) \approx \rho_{-}(\omega)$, for $T \gtrsim T_{c}$. We also confirm the results for the ground state masses, $M_{ \pm}$, from the exponential fits (see Table 2).

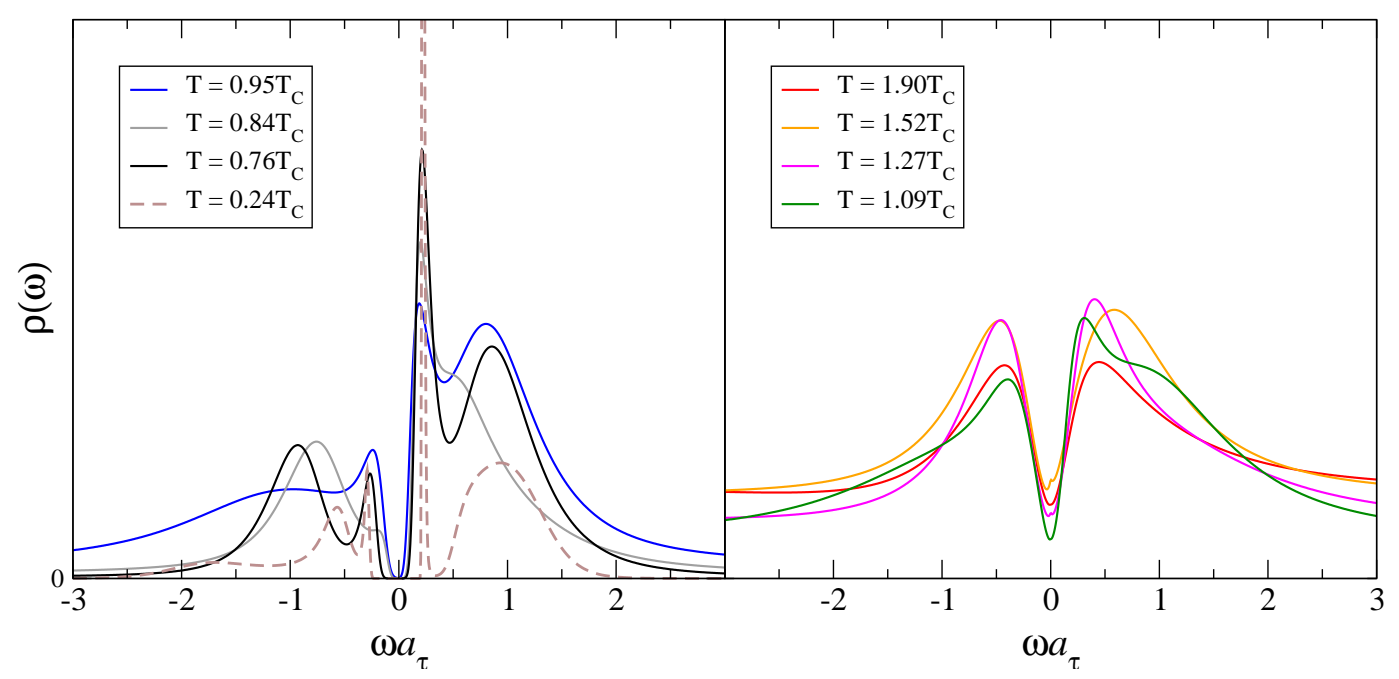

Figure 3: The spectral function $\rho(\omega)$ as a function of energy, $\omega$, with the left(right) pane showing temperatures below(above) $T_{c}$. The positive parity channel corresponds to $\omega>0$ and the negative parity to $\omega<0$, see Eq. 4.4.

We note that the results shown in this talk used smeared correlation functions, and thus it is crucial to confirm that no systematic effects are introduced by this procedure. These checks are performed in $[5,6]$.

\section{Conclusions}

We have analysed nucleon correlation functions to determine if parity doubling occurs for temperatures above $T_{c}$. We find evidence for this hypothesis by i) studying the correlators themselves, ii) fitting the correlators to exponentials, and iii) extracting the positive and negative parity spectral functions from an MEM analysis. Since deconfinement and chiral symmetry restoration are expected to occur around the same temperature $[18,19]$, the observed parity doubling can be understood from the restoration of chiral symmetry in the quark-gluon plasma.

\section{Acknowledgements}

This work used the DiRAC BlueGene/Q Shared Petaflop system at the University of Edinburgh, operated by the Edinburgh Parallel Computing Centre on behalf of the STFC DiRAC HPC Facility (www.dirac.ac.uk). This equipment was funded by BIS National E-infrastructure capital grant ST/K000411/1, STFC capital grant ST/H008845/1, and STFC DiRAC Operations grants 
ST/K005804/1 and ST/K005790/1. DiRAC is part of the National E-Infrastructure. We acknowledge the PRACE Grants 2011040469 and Pra05_1129, European Union Grant Agreement No. 238353 (ITN STRONGnet), the STFC grant ST/L000369/1, and All Souls College Oxford, HPC Wales, the Irish Centre for High-End Computing, the Leverhulme Trust, the Royal Society, STFC, and the Wolfson Foundation for support.

The authors would like to thank Seyong Kim, Maria Paola Lombardo, Mike Peardon, Sinéad Ryan and Don Sinclair, for useful comments, discussions and collaboration.

\section{References}

[1] R. Rapp and J. Wambach, Adv. Nucl. Phys. 25 (2000) 1 [hep-ph/9909229].

[2] C. E. DeTar and J. B. Kogut, Phys. Rev. Lett. 59 (1987) 399; Phys. Rev. D 36 (1987) 2828.

[3] S. Datta, S. Gupta, M. Padmanath, J. Maiti and N. Mathur, JHEP 1302 (2013) 145 [arXiv:1212.2927 [hep-lat]].

[4] I. Pushkina et al. [QCD-TARO Collaboration], Phys. Lett. B 609 (2005) 265 [hep-lat/0410017].

[5] G. Aarts, C. Allton, S. Hands, B. Jäger, C. Praki and J. I. Skullerud, Phys. Rev. D 92 (2015) 1, 014503 [arXiv: 1502.03603 [hep-lat]].

[6] G. Aarts, C. Allton, S. Hands, B. Jäger, C. Praki and J. I. Skullerud, in preparation

[7] C. Gattringer and C. B. Lang, "Quantum chromodynamics on the lattice," Lect. Notes Phys. 788 (2010) 1 .

[8] R. G. Edwards, B. Joó and H. W. Lin, Phys. Rev. D 78 (2008) 054501 [arXiv:0803.3960 [hep-lat]].

[9] H. W. Lin et al. [Hadron Spectrum Collaboration], Phys. Rev. D 79 (2009) 034502 [arXiv:0810.3588 [hep-lat]].

[10] A. Amato, G. Aarts, C. Allton, P. Giudice, S. Hands and J. I. Skullerud, Phys. Rev. Lett. 111 (2013) 172001 [arXiv:1307.6763 [hep-lat]].

[11] C. Allton et al., PoS LATTICE 2013 (2014) 151 [arXiv:1401.2116 [hep-lat]].

[12] G. Aarts, C. Allton, A. Amato, P. Giudice, S. Hands and J. I. Skullerud, JHEP 1502 (2015) 186 [arXiv:1412.6411 [hep-lat]].

[13] S. Güsken, U. Low, K. H. Mütter, R. Sommer, A. Patel and K. Schilling, Phys. Lett. B 227 (1989) 266.

[14] R. G. Edwards et al. [SciDAC and LHPC and UKQCD Collaborations], Nucl. Phys. Proc. Suppl. 140 (2005) 832 [hep-lat/0409003].

[15] J. Bulava et al., Phys. Rev. D 82 (2010) 014507 [arXiv:1004.5072 [hep-lat]].

[16] C. Praki and G. Aarts, Talk presented at Lattice 2015, Kobe, Japan, July 2015, PoS(LATTICE 2015) 182

[17] M. Asakawa, T. Hatsuda and Y. Nakahara, Prog. Part. Nucl. Phys. 46 (2001) 459 [hep-lat/0011040].

[18] S. Borsányi et al. [Wuppertal-Budapest Collaboration], JHEP 1009 (2010) 073 [arXiv:1005.3508 [hep-lat]].

[19] A. Bazavov, T. Bhattacharya, M. Cheng, C. DeTar, H. T. Ding, S. Gottlieb and R. Gupta et al., Phys. Rev. D 85 (2012) 054503 [arXiv:1111.1710 [hep-lat]]. 